\title{
Prototype of an opto-capacitive probe for non-invasive sensing cerebrospinal fluid circulation
}

Teemu Myllylä, Erkki Vihriälä, Matteo Pedone, Vesa Korhonen, Lukasz Surazynski, et al.

Teemu Myllylä, Erkki Vihriälä, Matteo Pedone, Vesa Korhonen, Lukasz Surazynski, Maciej Wróbel, Aleksandra Zienkiewicz, Jaakko Hakala, Hannu Sorvoja, Janne Lauri, Tapio Fabritius, Małgorzata Jędrzejewska-Szczerska, Vesa Kiviniemi M.D., Igor Meglinski, "Prototype of an opto-capacitive probe for non-invasive sensing cerebrospinal fluid circulation," Proc. SPIE 10063, Dynamics and Fluctuations in Biomedical Photonics XIV, 100630M (3 March 2017); doi: 10.1117/12.2251977

SPIE. Event: SPIE BiOS, 2017, San Francisco, California, United States 


\title{
Prototype of an opto-capacitive probe for non-invasive sensing cerebrospinal fluid circulation
}

\author{
Teemu Myllylä*a, Erkki Vihriälä̈, Matteo Pedone ${ }^{\mathrm{a}, \mathrm{b}}$, Vesa Korhonen ${ }^{\mathrm{c}, \mathrm{d}}$, Lukasz Surazynski ${ }^{\mathrm{a}}$, \\ Maciej Wróbel $^{\mathrm{e}}$, Aleksandra Zienkiewicz ${ }^{\mathrm{a}}$, Jaakko Hakala ${ }^{\mathrm{a}}$, Hannu Sorvoja ${ }^{\mathrm{a}}$, Janne Lauri ${ }^{\mathrm{a}}$, \\ Tapio Fabritius $^{\text {a }}$, Małgorzata Jędrzejewska-Szczerska ${ }^{\mathrm{e}}$, Vesa Kiviniemi ${ }^{\mathrm{c}, \mathrm{d}}$, Igor Meglinski ${ }^{\mathrm{a}}$ \\ ${ }^{a}$ University of Oulu, Optoelectronics and Measurement Techniques Unit, , Oulu, Finland; \\ ${ }^{\mathrm{b}}$ University of Oulu, Center for Machine Vision and Signal Analysis Unit, Oulu, Finland; ' Oulu \\ University Hospital, Medical Research Center (MRC), Oulu, Finland; ${ }^{\mathrm{d}}$ University of Oulu, Research \\ Unit of Medical Imaging, Physics and Technology, Oulu, Finland; ${ }^{\mathrm{C}}$ Gdańsk University of \\ Technology, Faculty of Elecronics, Telecommunications and Informatics, Department of Metrology \\ and Optoelectronics, Gdańsk, Poland
}

\begin{abstract}
In brain studies, the function of the cerebrospinal fluid (CSF) awakes growing interest, particularly related to studies of the glymphatic system in the brain, which is connected with the complex system of lymphatic vessels responsible for cleaning the tissues. The CSF is a clear, colourless liquid including water $(\mathrm{H} 2 \mathrm{O})$ approximately with a concentration of $99 \%$. In addition, it contains electrolytes, amino acids, glucose, and other small molecules found in plasma. The CSF acts as a cushion behind the skull, providing basic mechanical as well as immunological protection to the brain. Disturbances of the CSF circulation have been linked to several brain related medical disorders, such as dementia.

Our goal is to develop an in vivo method for the non-invasive measurement of cerebral blood flow and CSF circulation by exploiting optical and capacitive sensing techniques simultaneously. We introduce a prototype of a wearable probe that is aimed to be used for long-term brain monitoring purposes, especially focusing on studies of the glymphatic system. In this method, changes in cerebral blood flow, particularly oxy- and deoxyhaemoglobin, are measured simultaneously and analysed with the response gathered by the capacitive sensor in order to distinct the dynamics of the CSF circulation behind the skull. Presented prototype probe is tested by measuring liquid flows inside phantoms mimicking the CSF circulation.
\end{abstract}

Keywords: cerebrospinal fluid, brain, opto-capacitive probe, optical methods, capacitive sensing

\section{INTRODUCTION}

The glymphatic system (GS) of the brain is a waste clearance system [1] that utilizes cardiovascular pulsations to push cerebrospinal fluid (CSF) from perivascular space via astroglial cell aquaporin channels (AQP4), through brain tissue to eliminate soluble proteins and metabolites from the central nervous system (CNS). The GS is the counterpart of the lymphatic system elsewhere in the body. As a whole, it is responsible for flushing out toxins, metabolic waste products, soluble proteins and other harmful fluids from the body system into the CSF drainage [2]. In this process sufficient lymphatic and CSF circulation is essential which in turn is assumed to be driven by such as body movements, muscle contractions, breathing and, in particular, by the cardiovascular system where the heart beat and blood pressure (BP) indirectly maintain force of pressure in the lymphatic channels [3].

The glymphatic flow mechanisms are still not fully understood. Most of the studies are restricted to animal models or cadavers and the measurement methods exploited in these studies cannot be directly utilised for human in-vivo. One of the results from animal models indicate that particularly the reduction in glymphatic flow, due to closure or blockage in aquaporin channels in the brain, is linked with dementia related brain disorders [4]. Such blockages in aquaporin channels may occur also quickly for example because of brain edema resulting from traumatic brain injury.

*teemu.myllyla@oulu.fi

Dynamics and Fluctuations in Biomedical Photonics XIV, edited by Valery V. Tuchin, Kirill V. Larin,

Martin J. Leahy, Ruikang K. Wang, Proc. of SPIE Vol. 10063, 100630M · (c) 2017 SPIE

CCC code: $1605-7422 / 17 / \$ 18 \cdot$ doi: $10.1117 / 12.2251977$ 
Studies indicate that the GS is considerable more active during sleep [5], thus the GS may become insufficient also because of poor sleep quality [4]. Importantly, sleep studies should be conducted more since they are also directly linked to our daily lives. Failure of GS function might contribute to pathology in neurodegenerative disorders, traumatic brain injury and stroke [2]. In particular, recent studies indicate that insufficient GS causing amyloid accumulation in the brain, can be a major risk factor for development of neurodegenerative diseases, such as dementia and Alzheimer's disease (AD) $[4,6]$. Nevertheless, current methods to study mechanisms of the GS and glymphatic circulation in human brain are insufficient and in practice limited to positron emission tomography (PET) and magnetic resonance imaging (MRI) [3].

Based on the knowledge of light interaction [7,8,9] and dielectric properties [10] in tissue we propose a method of using capacitive and near-infrared spectroscopy (NIRS) sensing techniques simultaneously to measure average changes in cerebral hemodynamics and CSF circulation in the brain, assumed to reflect the function of the GS. Dielectric properties of tissue are well studied, however, utilization of a capacitive sensor for non-invasive CSF sensing are not reported. This paper studies the sensitivity of the presented opto-capacitive sensor for measuring average changes in its total measurement volume. Such technique, when further developed, could be potentially used for instance for measuring of GS activity during sleep. In the experimental measurements we used previously developed nanoparticles-free optical tissue phantoms, consisting of polydimethylosiloxane polymer and glycerol fabricated at Gdańsk University of Technology, Poland. The details of their fabrication and optical properties can be found in previous publications $[15,16]$.

\section{METHOD OF MEASUREMENT}

\subsection{Capacitive sensing method}

The basic operation principle of the constructed capacitance sensing method is commonly known and it is based on creating step excitations to the unknown capacitance $C_{X}$ by using a fixed resistor $R_{C}$ with known voltage values and measuring the time interval of each charge time constant which is dependent on $C_{X}$. The exact value of the unknown $C_{X}$, which is the capacitive field over the sensor plates, can be determined with the obtained results. In the current application (Fig. 1) the step excitations are created using an IC- timer (Texas Instruments LMC555) and the time interval of the responses is measured with a data acquisition device (National Instruments NI USB-6212).

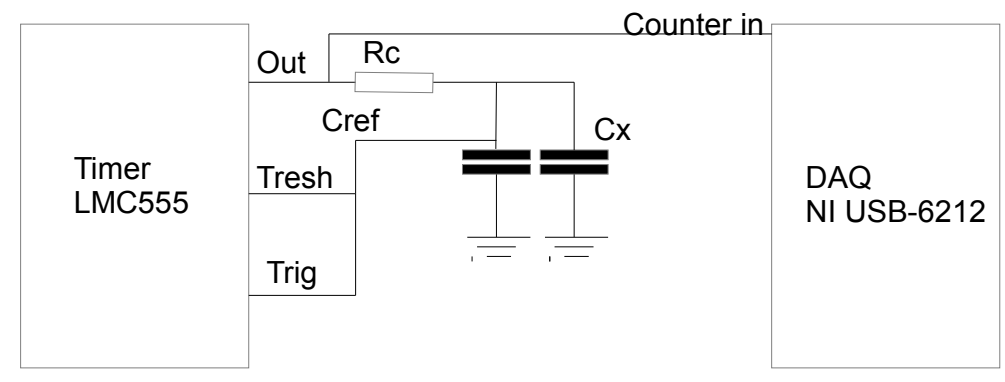

Figure 1. The basic construction of the capacitance measuring device. $\mathrm{V}_{\text {out }}, \mathrm{V}_{\text {tresh }}$ and $\mathrm{V}_{\text {trig }}$ voltages are presented in Fig.2.

LMC555 charges and discharges $C_{X}$ through $R_{C}=1 M \Omega$, with two fixed voltage levels (see Fig.1). For instance, $V_{\text {outh }}=$ $3.1 \mathrm{~V}$ (charging) and $\mathrm{V}_{\text {outl }}=0,2 \mathrm{~V}$ (discharging) creates step excitations whereas the charging and discharging periods are defined by timers having two threshold voltage levels of $\mathrm{V}_{\text {tresh }}=2,27 \mathrm{~V}$ and $\mathrm{V}_{\text {trig }}=1,14 \mathrm{~V}$. Now, if the voltage over $\mathrm{C}_{\mathrm{x}}$ is higher than $\mathrm{V}_{\text {tresh }}$ the discharging sequence starts, and respectively, if the voltage is lower than the $\mathrm{V}_{\text {trig }}$ the charging sequence starts. The time of high and low periods of the square wave (charging and discharging sequences), shown in Fig 2 , is read by a counter using $80 \mathrm{Mhz}$ clock frequency. 


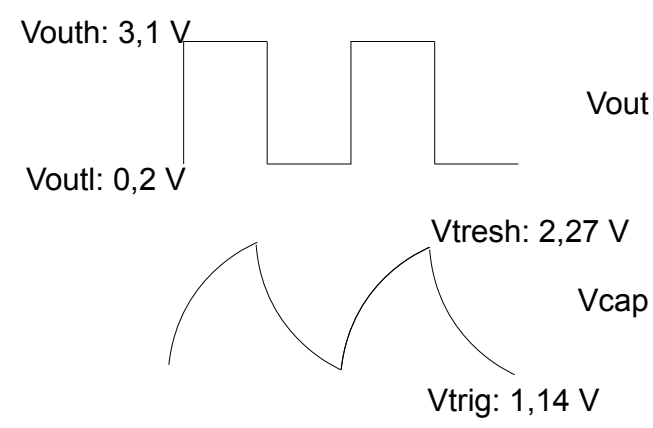

Vout: timers output (charging) voltage.

Vcap: voltage over the unknown capacitance.

Figure 2. Signals and voltages of the capacitance measuring device.

Further, the value of $\mathrm{C}_{\mathrm{x}}$ can be determined using the eq.1. if a reference capacitor $\left(\mathrm{C}_{\mathrm{ref}}=220 \mathrm{pF}\right.$ shown in Fig. 1$)$ is in parallel with $\mathrm{C}_{\mathrm{x}}$.

$$
C_{x}=\frac{T}{\left[\ln \left(\frac{V_{\text {tresh }}}{V_{\text {trig }}}\right)+\ln \left(\frac{V_{\text {outh }-V_{\text {trig }}}}{V_{\text {outh }} V_{\text {tresh }}}\right)\right] \times R_{C}}-C_{\text {ref }}
$$

In eq. (1), $\mathrm{T}=$ time periods of the square wave. $\mathrm{T}$ is $0,34 \mathrm{~ms}$ when $\mathrm{C}_{\mathrm{x}}$ is not connected which corresponds frequency of $2,9 \mathrm{kHz}$. In addition, the device uses a multiplexer (Texas Instruments CD4051) which enables selecting $\mathrm{C}_{\mathrm{x}}$ from eight different sources (Fig.3).

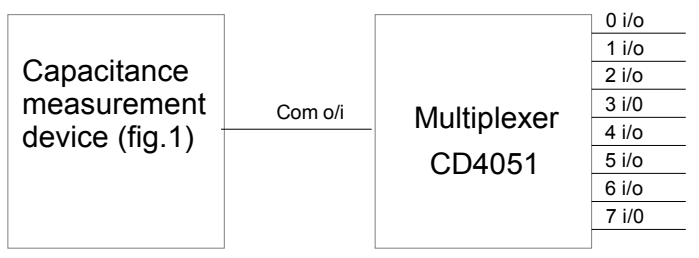

Figure 3. Capacitance measurement device connected to a multiplexer. $\mathrm{C}_{\mathrm{x}}$ can be selected from eight different sources (channels $0 \mathrm{i} / \mathrm{o}-7 \mathrm{i} / \mathrm{o}$ ).

\subsection{Simulations for capacitive sensing}

In order to validate the capacitive sensing technique described in the previous section, we ran simulations in a finite element analysis software (COMSOL Multiphysics v5.2a). Two simulations were performed.

The first, and more basic one, was aimed at demonstrating the feasibility of detecting small changes in volume of a liquid (water) with a capacitive sensor. The geometric setup is illustrated in Fig. 4a, left, and it depicts a scenario where a printed circuit board with a row of nine plates $(10 \mathrm{~mm}$ diameter, made of copper $)$ is placed under a polypropylene container. The container is partially filled with a layer of water, and the surrounding environment with air. The thickness of the layer of water is allowed to vary from $1 \mathrm{~mm}$ to $10 \mathrm{~mm}$, and such a variation is expected to produce a detectable change in the capacitance of the system. Although the row of capacitor plates was designed to detect spatially varying changes in capacitance, in this experiment the thickness of the water layer is constant across the container; for this reason, a voltage $(1 \mathrm{~V})$ was applied to five adjacent terminals, while the remaining four were grounded. The change in capacitance was measured and reported in Fig. 4b, right. In the right part of the plot where the slope of the capacitance curve is smaller, an increment of $1 \mathrm{~mm}$ of water level corresponds roughly to a change in capacitance of $100 \mathrm{fF}$, which is well within the minimum measurement accuracy of our instruments (10 fF). 
In the second simulation we generated a more challenging and realistic scenario, in which a capacitor with two parallel and coplanar copper plates (10 $\mathrm{mm}$ diameter) is placed in direct contact with a body whose internal structure simulates that of an average adult human head, with layers of skin $(3 \mathrm{~mm})$, skull $(7 \mathrm{~mm})$, and a layer of cerebrospinal fluid with variable thickness $(0 \mathrm{~mm}-2 \mathrm{~mm})$ surrounded by air, Fig. $4 \mathrm{~b}$. It is known that the relative permittivity of skin and bone is highly influenced by many factors (e.g. frequency, part of the body, wetness [14]). In our simulation we set the relative permittivity of skin and bone respectively to 40 and 15 . The relative permittivity of water was set to 80 . Analogously to the first simulation, we let the CSF layer to vary from 0 to $2 \mathrm{~mm}$ and we measured the respective capacitance. As the geometry of the capacitor has also an influence, we tested four configurations in which the (Hausdorff) distance between the capacitor plates was: $0.6 \mathrm{~mm}, 1.1 \mathrm{~mm}, 1.6 \mathrm{~mm}, 2.1 \mathrm{~mm}$, Fig. $4 \mathrm{a}$, right. For some configurations, an increment of 0.1 $\mathrm{mm}$ of the CSF corresponded to a minimum change of about $20 \mathrm{fF}$ in the capacitance, which is again measurable by our instruments.
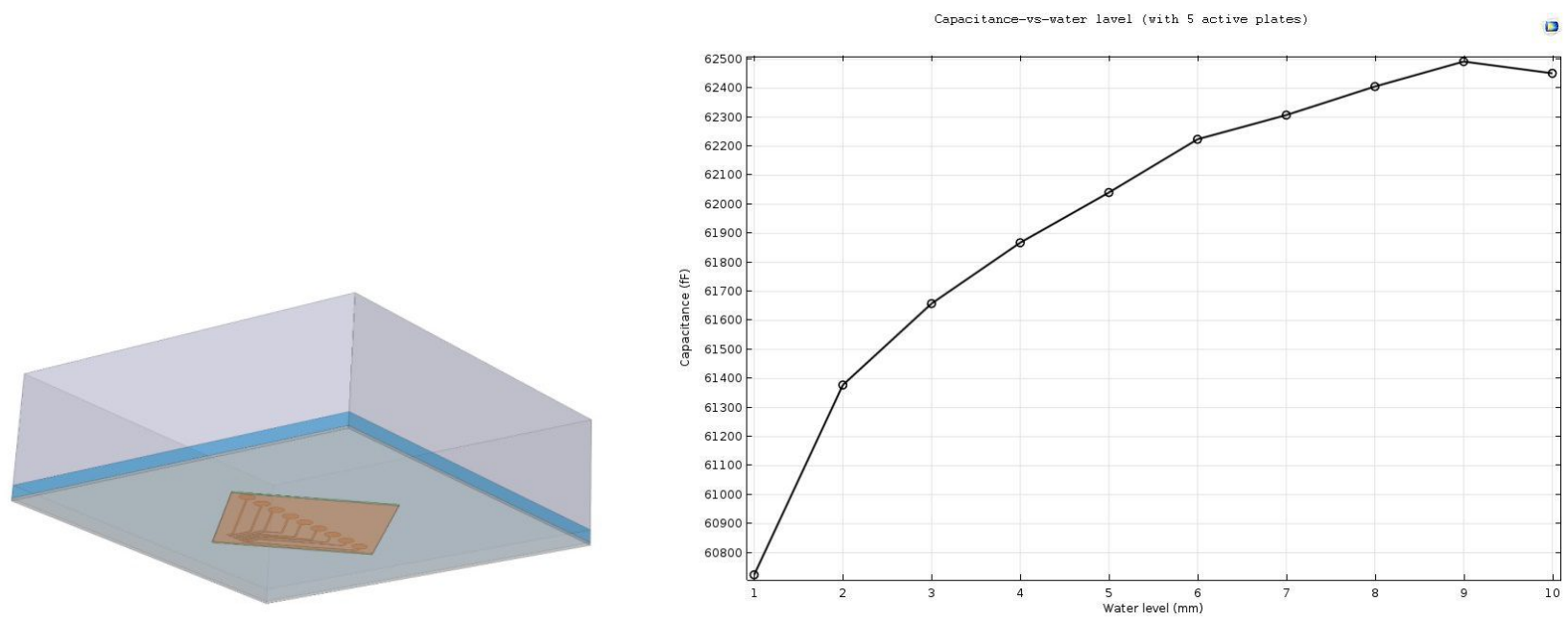

Figure 4a. Geometric setup for the first simulation. A row of capacitor plates is placed under a polypropylene box containing a variable amount of water. The change in volume of water causes a change in capacitance which can be measured by the sensor (left). Change in capacitance as a function of the level of water (right).
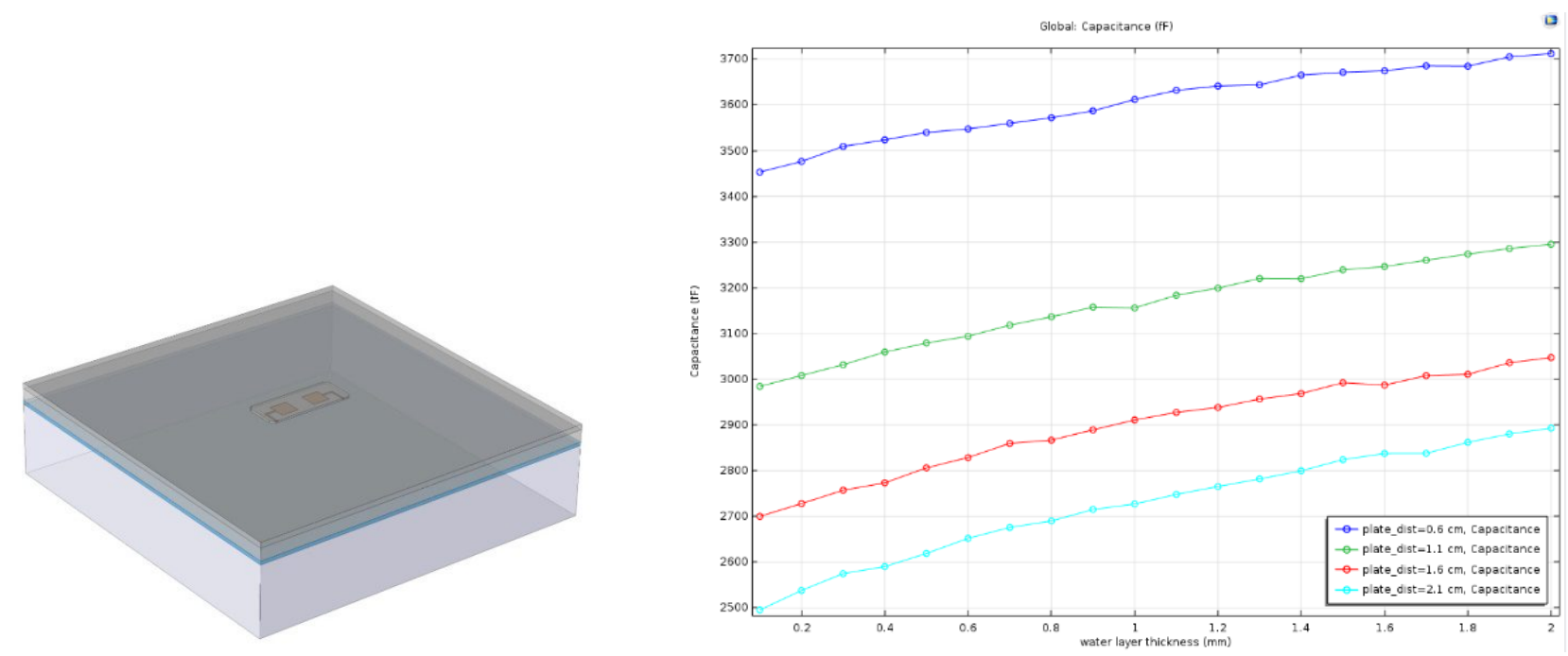

Figure 4b. Geometric setup for the second simulation. A capacitor is placed on top of a human head with layers of skin, skull bone, and cerebrospinal fluid (left). The change in volume of the CSF is plotted against the change in capacitance for different configurations of the capacitor (right). 


\subsection{Optical method for measuring hemoglobin and blood volume}

The detection and the quantification of the chromophore concentrations in the brain cortex is based on known absorbance peaks and isosbestic points of the corresponding chromophores mostly in the spectrum range between 650 $\mathrm{nm}$ and $1000 \mathrm{~nm}$, where light attenuation in tissue is low enough, enabling light penetration deeper into tissue, to reach the cerebral cortex of the brain [11]. There are two primary chromophores that are of our interests in NIRS studies: oxyhemoglobin $(\mathrm{HbO})$, deoxyhemoglobin $(\mathrm{Hb})$. In addition, for water there are absorbance peaks approximately at 980 $\mathrm{nm}$ and at $1450 \mathrm{~nm}$ that are possible to sense using a single photodiode also when simultaneously quantifying $\mathrm{HbO}$ and $\mathrm{Hb}$ [17], for instance, an InGaAs PIN photodiode G10899 by Hamamatsu with a spectrum range of approximately 500 $\mathrm{nm}$ to $1700 \mathrm{~nm}$. In particular, we can determine total hemoglobin (HbT) by NIRS, which reflects changes of blood volume in the measurement volume. This measure can be potentially utilized in distinguishing blood volume and water volume when combined with the capacitive sensing. For this, mathematical models and inverse problem analysis methods are developed, however, these are not discussed here. In this study, we focused on the capacitive sensing thus current NIRS probe features only two wavelengths $660 \mathrm{~nm}$ and $830 \mathrm{~nm}$ that are used for quantifying $\mathrm{Hb}, \mathrm{HbO}$ and $\mathrm{HbT}$.

\section{RESULTS}

In our experiments we studied water sensitivity of the opto-capacitive probe by using two methods. In the first experiment, the presented capacitive sensor was tested using the same geometric setup and method as in the simulation (see Figure 4a, left). Fig. 5, on the right, shows average response of the capacitive sensor which is comparable to the simulation result, shown on the left. As can be seen, both simulation and real measurement show that small changes in water volume can be detected.
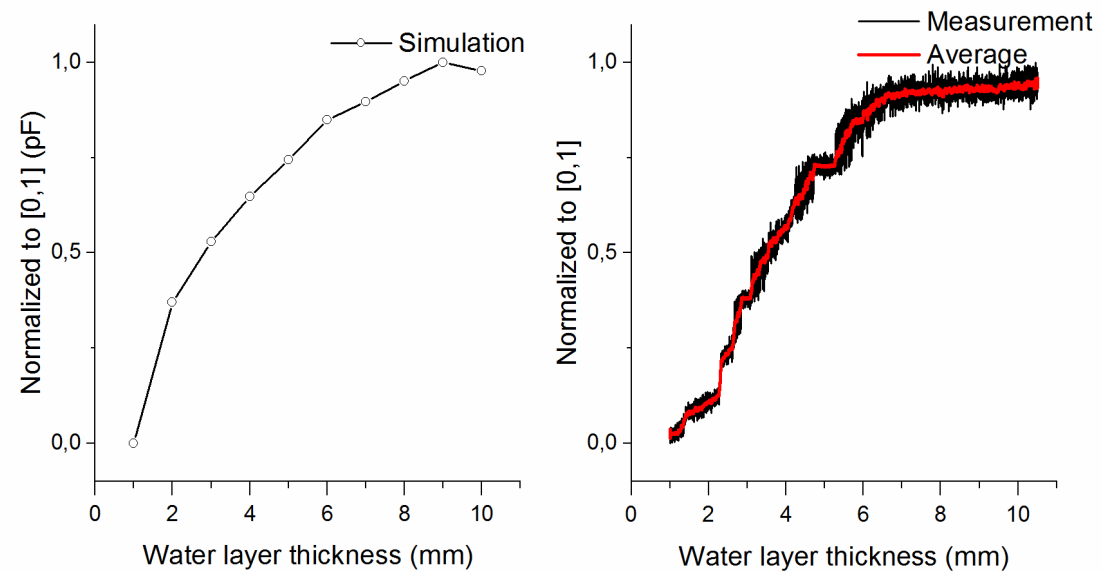

Figure 5. Capacitive sensor can detect small changes in volume of water. The geometric setup is illustrated in Fig. 4a, where a printed circuit board with a row of nine plates $(10 \mathrm{~mm}$ diameter, made of copper) is placed under a polypropylene container. In the container, the thickness of the layer of water was varied from $1 \mathrm{~mm}$ to $10 \mathrm{~mm}$.

Second experiment was done using a multilayered phantom, consisting of four tissue-mimicking layers (skin, skull, grey and white matter) which were fabricated to resemble the optical properties of forehead tissues [18]. Thicknesses of rectangular $(9.5 \times 9.5 \mathrm{~cm})$ tissue layers were: skin $(3 \mathrm{~mm})$, skull $(7 \mathrm{~mm})$, grey matter $(4 \mathrm{~mm})$ and white matter $(10 \mathrm{~mm})$. The phantoms were created according to previously established procedures [15]. In short, the PDMS consisting of a base material and a curing agent were mixed together (10 parts of base to 1 part of curing agent) with glycerol in the amounts ranging from 1 to 10 parts, to achieve the scattering coefficient most similar to that of the tissues. The influence of absorbing agent was not studied in this case as we focus solely on the scattering-related optical phenomena.

In the measurements, the CSF flow was simulated by injecting $5 \mathrm{ml}$ of water between skull and grey matter layers. After that the water stayed and freely spread between these layers, mimicking CSF. In the phantom, dielectric properties of tissue were not taken into account. Thus, in this case, we could only study if the capacitive sensor is capable of detecting small changes in water volume and flow at a measurement distance of $10 \mathrm{~mm}$, which is approximately the distance between skin surface and CSF layer. In the optical probe wavelengths of $660 \mathrm{~nm}$ and $830 \mathrm{~nm}$ are used and their sensitivity to water in the CSF layer was experimented. Naturally, if effect of blood in the second experiment was taken 
into account, these wavelengths would be probably more affected by it than CSF. However, it is interesting to notice that changes of water volume in CSF layer seem to affect these wavelengths, as Fig. 6 shows.

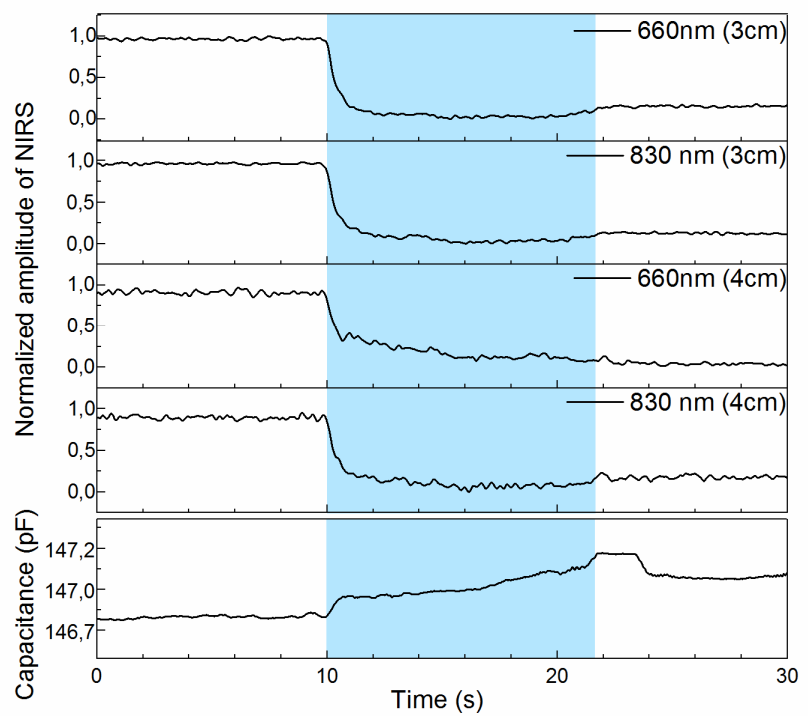

Figure 6. Effect of water injection $(5 \mathrm{ml})$ between skull and grey matter layers, mimicking CSF flow. The moment of injections is highlighted. As can be seen, both sensing techniques seem to be sensitive to small water volume changes in the CSF layer. In the optode, two source-detector distances $(3$ and $4 \mathrm{~cm}$ ) were used simultaneously where $3 \mathrm{~cm}$ distance seems to have slightly better sensitivity to CSF. However, in this experiment effect of blood is not taken into account.

\section{DISCUSSION}

Considering the capacitance measuring device the bandwidth and the resolution of the device could be adjusted by changing the charging resistor value $\left(\mathrm{R}_{\mathrm{c}}\right.$, Fig.1). Increasing the resistor value would lower the bandwidth and increase the resolution. Different resistor values were tested and it was found out that relatively high bandwidths also yielded highest signal-noise ratios, even if the resolution was lower. The measurement related to the simulation was performed with the resistor value $1 \mathrm{M} \Omega$ corresponding oscillation frequency being about $2.9 \mathrm{kHz}$ (Fig.2). Considering the signalnoise ratio it was also found out that measuring environment had much effect on it: while the measurements were executed in the water surface the noise increased dramatically comparing to the dry environment, even if the capacitor plates had no direct contact with the water. The devices structure (Fig.1) caused the polarity of the $\mathrm{C}_{\mathrm{x}}$ being the same (the direction of the voltage is the same all the time) and the modification that allowed polarity changes made minor improvement to the devices signal-noise ratio.

Sensitivity measurements of the opto-capacitive probe were performed without taking into account blood flow in skin and grey matter of the brain which both are assumed to have an effect also on the capacitive response. Our next step is to study these related characteristics in particular. Additionally, in the optical part of the probe we aim to use also wavelengths near the absorbance peaks of water. Presented prototype of the opto-capacitive probe certainly still requires further development and validation of its accuracy. Being small of size as well as potentially suitable for continuous long term measurement purposes, for instance during sleep, it may possibly be used in near future as a tool for studies of GS.

\section{ACKNOWLEDGEMENTS}

This study was partially funded by European Regional Development Fund, coordinated by Centre for Health and Technology, Oulu, Finland; and by Academy of Finland \& JAES-grants. M.S. W. and M. J-Sz acknowledge the support of the National Science Center, Poland, grant no. 2016/20/T/ST7/00380, as well as DS Programs of the Faculty of Electronics, Telecommunications and Informatics, Gdańsk University of Technology. EU COST BM1205 Action "Skin Cancer Laser Imaging". 


\section{REFERENCES}

[1] Louveau, Antoine, et al. "Structural and functional features of central nervous system lymphatic vessels." Nature (2015).

[2] Raper, Daniel, Antoine Louveau, and Jonathan Kipnis. "How Do Meningeal Lymphatic Vessels Drain the CNS?." Trends in Neurosciences 39.9 (2016): 581-586.

[3] Kiviniemi, Vesa, et al. "Ultra-fast magnetic resonance encephalography of physiological brain activityGlymphatic pulsation mechanisms?." Journal of Cerebral Blood Flow \& Metabolism (2015): $0271678 X 15622047$.

[4] Ooms, Sharon, et al. "Effect of 1 night of total sleep deprivation on cerebrospinal fluid $\beta$-amyloid 42 in healthy middle-aged men: a randomized clinical trial." JAMA neurology 71.8 (2014): 971-977.

[5] Xie, Lulu, et al. "Sleep drives metabolite clearance from the adult brain." science 342.6156 (2013): 373-377.

[6] Spies, Petra E., et al. "Reviewing reasons for the decreased CSF Abeta42 concentration in Alzheimer disease." Front Biosci 17 (2012): 2024-34.

[7] Korhonen, Vesa O., et al. "Light propagation in NIR spectroscopy of the human brain." IEEE Journal of Selected Topics in Quantum Electronics 20.2 (2014): 289-298.

[8] Tuchin, Valery V. "Tissue Optics, Light Scattering Methods and Instruments for Medical Diagnostics." (2015).

[9] Shi, Lingyan, et al. "Transmission in near-infrared optical windows for deep brain imaging." Journal of biophotonics 9.1-2 (2016): 38-43.

[10] Axt, Brant, Song Zhang, and Rajesh Rajamani. "Wearable Coplanar Capacitive Sensor for Measurement of Water Content-A Preliminary Endeavor." Journal of Medical Devices 10.2 (2016): 020953.

[11] Tuchin V.V.,"Tissue Optics: Light Scattering Methods and Instruments for Medical Diagnosis,” 3rd ed., PM 166. Bellingham, WA, USA: SPIE Press (2015).

[12] Bhutta, M. R. and Keum-Shik Hong,"Water correction algorithm to increase the signal strength of oxy and deoxy-hemoglobin in near-infrared spectroscopy signals," Industrial Technology (ICIT), 2014 IEEE International Conference on, 589-593 (2014).

[13] Tsuji M., Naruse H., Volpe J. \& Holtzman D.,"Reduction of Cytochrome aa3 Measured by Near-Infrared Spectroscopy Predicts Cerebral Energy Loss in Hypoxic Piglets," Pediatr Res 37(3): 253-259 (1995).

[14] Gabriel C., "Compilation of the Dielectric Properties of Body Tissues at RF and Microwave Frequencies," King's College London (United Kingdom), Dept. of Physics (1996).

[15] Wróbel, M. S., Popov, A. P., Bykov, A. V., Tuchin, V. V.., Jędrzejewska-Szczerska, M., "Nanoparticle-free tissue-mimicking phantoms with intrinsic scattering," Biomedical Optics Express 7(6), 2088 (2016).

[16]Feder, I., Wróbel, M., Duadi, H., Jędrzejewska-Szczerska, M., Fixler, D., "Experimental results of full scattering profile from finger tissue-like phantom," Biomedical Optics Express 7(11), 4695-4701 (2016).

[17] Myllylä, T., Korhonen, V., Kiviniemi, V., Tuchin, V, "Experimental studies with selected light sources for NIRS of brain tissue: quantifying tissue chromophore concentration", Proc. SPIE 9305, Optical Techniques in Neurosurgery, Neurophotonics, and Optogenetics II, 93051S (March 10, 2015), doi:10.1117/12.2076954.

[18] Wróbel, M. S., Popov, A. P., Bykov, A. V., Kinnunen, M., Jędrzejewska-Szczerska, M., Tuchin, V. V., "Multilayered tissue head phantoms for noninvasive optical diagnostics," J. Innov. Opt. Health Sci. 8(3), 1541005 (2015). 